Check for updates

Cite this: RSC Adv., 2017, 7, 51944

Received 7th September 2017 Accepted 2nd November 2017

DOI: 10.1039/c7ra09954a

rsc.li/rsc-advances

\section{Blended additive manipulated morphology and crystallinity transformation toward high performance perovskite solar cells $\uparrow$}

\author{
Dan Liu, $\hbar^{\mathrm{a}}$ Chongwen Li, $\dot{+}^{\mathrm{bc}}$ Cuiping Zhang, ${ }^{\mathrm{b}}$ Zaiwei Wang, ${ }^{\mathrm{b}}$ Huawei Zhang, ${ }^{\mathrm{d}}$ \\ Jintao Tian*a and Shuping Pang (iD *b
}

\begin{abstract}
The efficiency of a perovskite solar cell is highly affected by the crystal quality and morphology of its perovskite layer. A facile strategy of using blended organic additives to regulate the film's crystallinity and coverage is studied in this work. The coordination behavior and sublimation temperature of the additives are the essential characteristics which determine the quality of the perovskite films. By introducing a certain amount of $\mathrm{MACl}$, a kind of crystallinity-preferred additive, to partially replace MAAc, a coverage-preferred additive, the film's crystallinity has been obviously improved at no expense of coverage, which results in a lower trap density, higher efficiency and better stability.
\end{abstract}

\section{Introduction}

Organic-inorganic metal halide perovskites (OIMHPs) have drawn extensive attention due to their rapidly increasing power conversion efficiency (PCE) to over $22 \%$ within only 7 years..$^{1-3}$ Except for the superior optoelectronic properties of the perovskite layer, the feasibility of the solution process also makes them promising for both laboratory studies and industrial production. ${ }^{4-6}$ In ideal perovskite solar cell (PSCs) devices, the perovskite layer should be both highly uniform and with high crystallinity to reduce energy loss during carrier diffusion and extraction. $^{7-9}$ A stoichiometric precursor solution which contains equal molar amounts of lead iodine $\left(\mathrm{PbI}_{2}\right)$ and methylammonium iodide $\left(\mathrm{CH}_{3} \mathrm{NH}_{3} \mathrm{I}_{3}\right.$ or MAI) dissolved in an organic solvent such as $N, N$-dimethylformamide (DMF), butyrolactone (GBL) or dimethylsulfoxide (DMSO) is the most typical precursor solution prescription. Because of the strong coordination bonds between solvent (SV) and $\mathrm{PbI}_{2}$, the perovskite precursor presents its composition mostly in the form of SV$\mathrm{PbI}_{2}-\mathrm{MAI}, \mathrm{SV}-\mathrm{PbI}_{2}-\mathrm{SV}$ and $\mathrm{MAI}-\mathrm{PbI}_{2}-\mathrm{MAI}$ complexes in solution. ${ }^{\mathbf{1 0}-13}$ The retarded nucleation rate and heterogeneous growth nature of the coordination complexes during the

${ }^{a}$ School of Materials Science and Engineering, Ocean University of China, Qingdao 266100, P. R. China.E-mail: jttian@ouc.edu.cn

${ }^{b}$ Qingdao Institute of Bioenergy and Bioprocess Technology, Chinese Academy of Sciences, Qingdao 266101, P. R. China. E-mail: pangsp@qibebt.ac.cn

${ }^{c}$ Department of Physics and Astronomy, Wright Center for Photovoltaics Innovation and Commercialization, The University of Toledo, Toledo, USA

${ }^{d}$ College of Chemical and Environmental Engineering, Shandong University of Science and Technology, Qingdao 266590, P. R. China

$\dagger$ Electronic supplementary information (ESI) available. See DOI: 10.1039/c7ra09954a

\$ These authors contributed equally to this work. evaporation of non-coordinated solvent eventually lead to the formation of bulk rod-like morphologies. As the following removal of the residual coordinated solvent molecules is a solid state decomposition reaction, it still maintains very poor film morphology. ${ }^{13}$

Taking these into consideration, to replace the SV molecules coordination by introduction of stoichiometric ratio of additive is proved to be an effective way to enhance perovskite crystal quality. The recipes of $\mathrm{PbI}_{2}: \operatorname{MACl}(1: 3), \mathrm{PbI}_{2}: \mathrm{MAI}: \mathrm{MACl}$ $(1: 1: 1)$, and $\mathrm{PbI}_{2}: \mathrm{PbCl}_{2}:$ MAI $(1: 1: 4)$ recipes have developed to synthesis the $\mathrm{MAPbI}_{3-x} \mathrm{Cl}_{x}$ perovskite films. ${ }^{14-17}$ Other precursor solutions, such as employing MAAc directly as additive in the precursor solution, could deliver very smooth and compact films, whereas the crystal size is much smaller, normally resulting in reduced photoluminescence intensity and lifetime. ${ }^{\mathbf{1 8 , 1 9}}$ Besides, other solvent additives, such as 1-chloronaphthalene, also can enhance the film uniformity via interacting with $\mathrm{Pb}^{2+}$ to form a chelate complex. ${ }^{20}$ In many single additive related cases, either the film uniformity or the crystallinity could be obviously improved in comparison with nonadditive $\mathrm{PbI}_{2}$ : MAI $(1: 1)$ system. Generally, there is an offset of the film uniformity and crystallinity because they are conflicting as the high uniform film acquires a fast nucleation process, which normally reduces the time for grain growth and produces large amount of grain boundary defects in the films.

Herein, we employ a strategy of blended-additives engineering to regulate perovskite film morphology and crystallinity, in which MAAc ensures film smoothness and coverage, and MACl facilitates perovskite film crystallinity. The component of the precursor in our method is $\mathrm{MAPbI}_{3}$ with equal molar of blended $x$ MAAc $\cdot(1-x) \mathrm{MACl}$ additives. By appropriate change the ratio of these two components, perovskite film crystallinity and morphology will be regulated accordingly. 
Benefiting from the modified film properties, the optimized device in this work shows lower trap density, improved stability and better efficiency.

\section{Result and discussion}

Fig. $1 \mathrm{~A}-\mathrm{C}$ shows the typical morphology of $\mathrm{MAPbI}_{3}$ films using MAAc, MACl and blended additives respectively. As can be seen in Fig. $1 \mathrm{~A}, \mathrm{MAPbI}_{3}$ film with MAAc additive (MAPI-MAAc) shows very smooth and compact film morphology, in which with small crystals of about $150 \mathrm{~nm}$. As for the film with $\mathrm{MACl}$ additive (MAPI-MACl), the size of perovskite crystals distinctly enhanced to about $600 \mathrm{~nm}$, but with apparent pinholes between them (Fig. 1B). After replacing certain amount of MACl by MAAc, the fabricated sample shows enhanced film crystallinity at no expense of film coverage as shown in Fig. 1C. The feasibility of this regulating strategy is evidenced in Fig. S1 $\uparrow$ by a series of blended organic additives (BOAs) films. When MACl component increases (MAAc amount decreases), the perovskite crystal size correspondingly shows an increases tendency. However, excessive amount of MACl in the component will result in the shrink of the perovskite crystals which destroys the film coverage. When $x$ reaches to 0.8 , the fabricated films exhibit optimized properties in the consideration of both film coverage and crystal size, which can be seen in Fig. 1C. Fig. 1D shows the UV-vis absorption spectra of the three films. All the three films show very similar absorption lines which indicate their similar optical properties. The intensity of MAPI-BOAs is slight stronger compared to the other two films in the region below $700 \mathrm{~nm}$, which is mainly attributed to improvement of the uniformity compared with the MAPI-MACl film. The X-ray diffraction (XRD) patterns for these three films are also measured to compare their phase composition and crystal property. As can be seen in
Fig. 1E, all these three films show characteristic diffraction peaks of $2 \theta=14.0^{\circ}$ and $28.4^{\circ}$, which indicate their complete conversion to $\mathrm{MAPbI}_{3}$ perovskite from precursor solutions. The peaks intensity of MAPI-MACl and MAPI-BOAs films however are much stronger than that of MAPI-MAAc film, which indicates their preferential crystal growth along (110) faces and higher crystallinity. From what have been shown above, the MAPI-BOAs film with $80 \%$ MAAc and $20 \%$ MACl still retains fullcoverage morphology but with obviously improved crystallinity.

Numerous additive-related works have been done before, but the properties of the fabricated films differ as the additives vary in these works and the mechanism is still not very clear. ${ }^{21,22}$ It's been known that the crystallization process can be divided into two simultaneous processes, which are nucleation and crystal growth. ${ }^{23}$ The distinct morphology and crystal quality of these three films should be ascribed to their very different crystallization process. We hereafter investigated their crystallization process from the stage of precursor solutions to final perovskite films, which should be of great importance to the understanding of their morphology distinction. Perovskite precursor solutions, due to their strong coordination effects between molecules, are deemed as colloidal solutions which contain colloidal dispersions dispersed in them. ${ }^{10}$ Fig. $2 \mathrm{~A}-\mathrm{D}$ shows the dynamic light scattering measurements of the three precursor solutions with different additives and pristine $\mathrm{MAPbI}_{3}$ precursor solution, to evaluate their colloidal particle size distribution. In Fig. 2A of pristine $\mathrm{MAPbI}_{3}$ precursor solution, the particle size distribution peak is at around $512 \mathrm{~nm}$, which indicates its well-defined colloidal property. While for the other three solutions with additives, the particle sizes decrease to varying extents. For MAPI-MACl sample, the particle sizes is about $412 \mathrm{~nm}$ (Fig. 2B), and after the blending of MAAc, the size decrease to 376 and $236 \mathrm{~nm}$ respectively for MAPI-BOAs
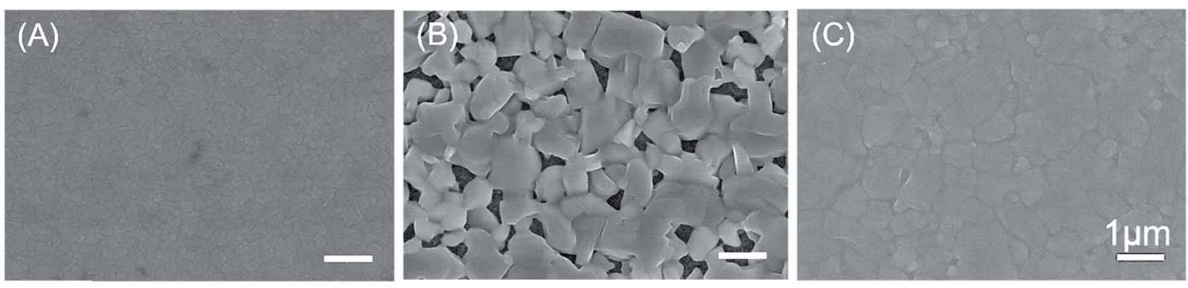

(D)

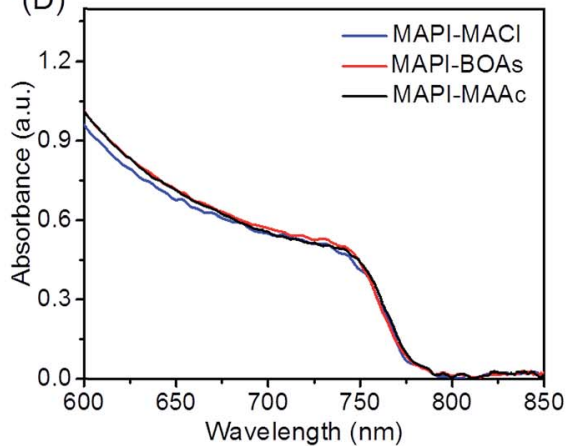

(E)

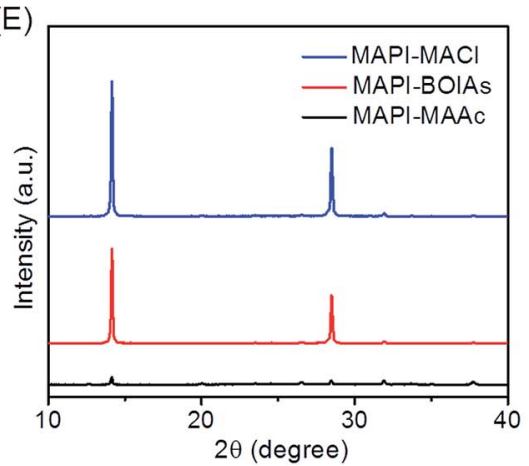

Fig. 1 (A-C) SEM images of MAPI-MAAC, MAPI-BOAs and MAPI-MACl perovskite films. (D and E) The corresponding UV-vis absorption spectra and XRD patterns of the three samples. 

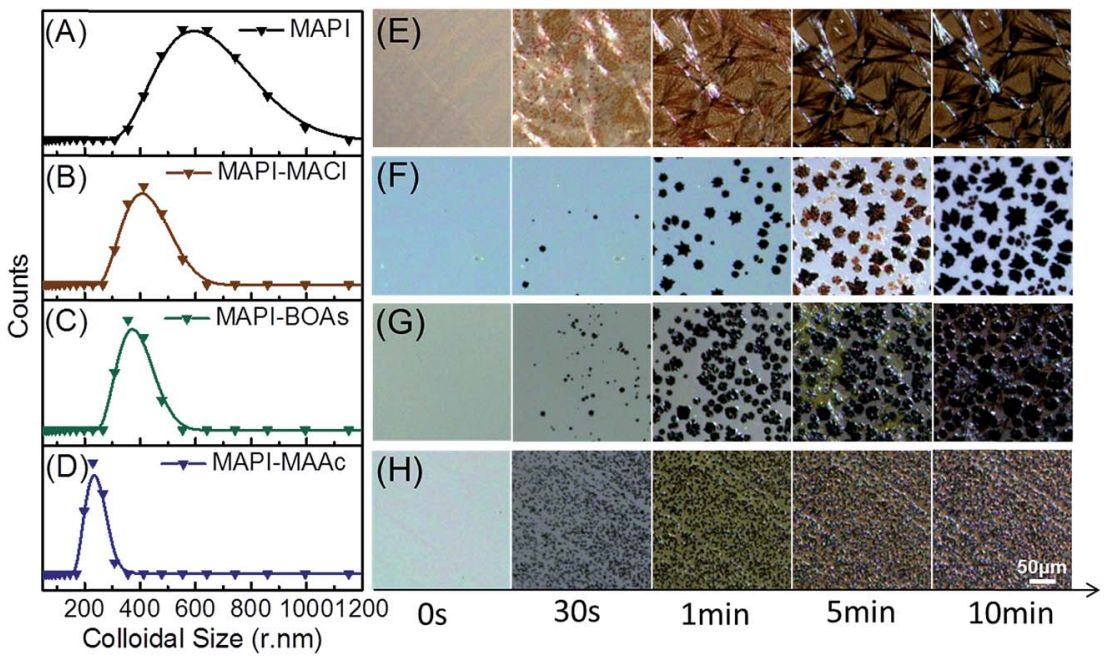

Fig. 2 (A-D) Colloidal particles size distribution of MAPI, MAPI-MACl, MAPI-BOAs, MAPI-MAAc precursor solutions. (E-H) In situ microscopy images of the crystallization process of the three films fabricated from MAPI, MAPI-MACl, MAPI-BOAs, MAPI-MAAc precursor solutions.

(Fig. 2C) and MAPI-MAAc (Fig. 2D) samples. This is because the excess $\mathrm{I}^{-} / \mathrm{Ac}^{-}$anions/anion groups from additives have strong coordination effects which boost the maximum coordination state of the obtained $\left[\mathrm{PbI}_{6-x} \mathrm{Ac}_{x}\right]^{4-}(x=0-6)$ quasi-octahedron. ${ }^{10}$ Besides, the ionized organic component $\left(\mathrm{MA}^{+}\right)$will insert in between the $\left[\mathrm{PbI}_{6-x} \mathrm{X}_{x}\right]^{4-}$ frameworks, cutting them into small ones. $^{8,24,25}$ Especially, the MAPI-MAAc solution shows the smallest particle size compared to others, this however should be associated with the difference of coordination bond strength between them, which determines their intermolecular distances. Other factors, such as the size difference of $\mathrm{I}^{-}$and $\mathrm{Ac}^{-}$and their corresponding $\left[\mathrm{PbI}_{6-x} \mathrm{Ac}_{x}\right]^{4-}$ frameworks, should also be taken into consideration. Upon spinning coating the precursor solution on substrate, the nucleation and grain growth processes happens.

Fig. 2E-H shows the in situ microscopy of the crystallization process of $\mathrm{MAPbI}_{3}$ from the precursor solutions with/without additives. The samples are prepared on $\mathrm{TiO}_{2}$ scaffold by blading and low temperature annealing treatment is employed to prolong this process for observation. Although the testing condition is different from the normal treating process of perovskite films, the visible nucleus and growth processes are helpful for us to understand the additives' functions. At the origin stage, the precursor solutions are spread evenly on the substrates. At the first stage of 30 seconds, all the precursors with/without additives start to nucleate but with different nucleus density. For MAPI-MACl film, very few nucleation centers are formed on the surface. The number of nucleation sites grows when the additive changes to BOAs. For MAPI-MAAc film, the nucleation density grows prominently, and these crystal nucleus scatters averagely on the glass, but the size of them decrease compared to that of the former two. The small size distribution of the intermediate phase in mother solution should contribute to the size contraction of the crystal nucleus precipitating on substrate and this is one aspect from where, the small size of MAPI-MAAc perovskite crystals forms. For MAPI film, very dense nuclei are formed on the substrate, and rod-like crystals seems to appear at this very early stage. At about 1 minute, more nucleuses are grown on the substrate for these films, which highly follow the characteristics of their former stages. Besides, the crystal size grows larger for MAPIMACl and MAPI-BOAs samples, but not obvious for MAPIMAAc and MAPI films. When it comes to 5 minutes, the nucleuses continue to grow bigger only for MAPI-MACl and MAPI-BOAs films. At the last stage of 10 minutes, sparse and large crystals are grown on the surface of MAPI-MACl film, while for MAPI-MAAc film, very compact and small crystals are arranged. For MAPI-BOAs film, the crystal density is larger than that of MAPI-MACl film, and the crystal size shows no obvious reduction. However, for MAPI film, the film morphology shows no change at this stage. The difference in nucleation density and crystal size of these films at the same stage is associated with the properties of their different intermediates. MAAc is a kind of volatile matter, while MACl has much higher volatility as can be seen in Fig. S3. $\dagger$ The low sublimation temperature of MAAc can both accelerate nucleation rate and form higher nucleation density. However, the perovskite crystals size in MAPI-MAAc film (Fig. 1A and $2 \mathrm{H}$ ) is too small which will introduces large number of grain boundaries, and they are where the defects usually locates. ${ }^{9}$ By incorporating $20 \%$ of $\mathrm{MACl}$, the volatility of the intermediate phase is increased, which regulates the nucleation rate and density. By precisely control the ratio of the blended additives, the MAPI-BOAs film can possess both full-coverage morphology and high quality perovskite crystals at the same time.

As can be concluded from what have been discussed above, the coverage and crystallinity of the films by MACl blending is enhanced. To further assess the film quality by MACl blending, a series of optoelectronic properties are measured. Fig. 3A shows the time-resolved photoluminescence (TRPL) decay measurements of MAPI-MAAc and MAPI-BOAs films (on glasses). The curves can be well fitted with a biexponential function containing both slow and long lifetimes, which are $\tau_{1}$ and $\tau_{2}$ respectively. We assign $\tau_{1}$ as the decay which is closely 

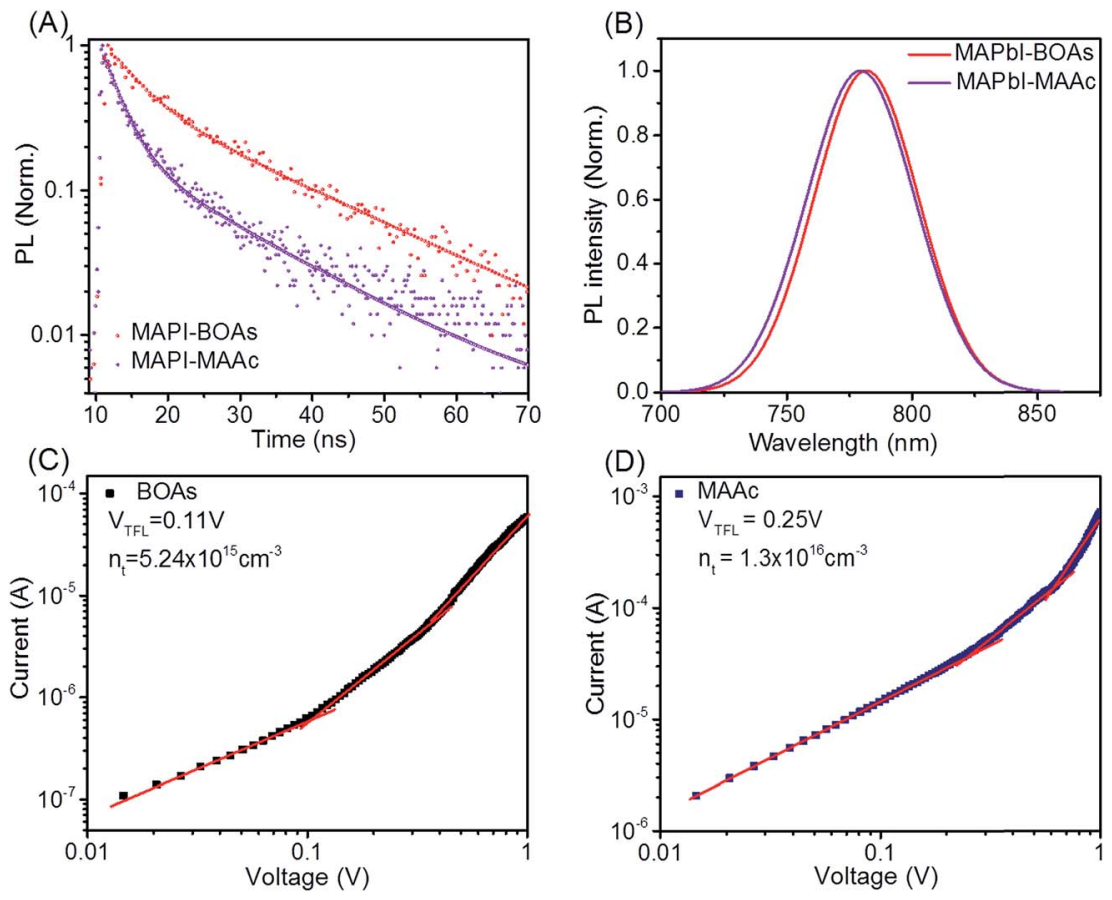

Fig. 3 (A) Time-resolved photoluminescence decay for MAPI-MAAc, MAPI-BOAs perovskite films. (B) Normalized photoluminescence spectra of MAPbI-MAAc, MAPbI-BOAs perovskite films. ( $C$ and D) I- $V$ curves of MAPI-MAAc and MAPI-BOAs devices. The devices employ FTO/com-TiO 2 I perovskite/PCBM/Ag configuration. The $V_{\mathrm{TFL}}$ is obtained from linearly fitted logarithmic $I-V$ plot, and utilized for calculating the trap densities.

related to the non-radiative recombination by the traps on film surface and grain boundaries, and $\tau_{2}$ as the decay of radiative recombination from bulk perovskite which is associated with bulk carrier recombination. ${ }^{26}$ For MAPI-MAAc sample, $\tau_{1}$ is calculated to be $2.5 \mathrm{~ns}$. While for MAPI-BOAs sample $\tau_{1}$ increases to $4.3 \mathrm{~ns}$, which indicates its fewer non-radiative recombination on the film surface and between grain boundaries. MAPI-BOAs film shows longer $\tau_{2}$ of $18.9 \mathrm{~ns}$ compared to $15.0 \mathrm{~ns}$ of MAPI-MAAc sample, indicating the recombination in the bulk film is also efficiently reduced. For comparison, the TRPL decay of MAPb-MACl film was also measured as shown in Fig. S5A. $\dagger$ The calculated $\tau_{1}$ and $\tau_{2}$ are $5.6 \mathrm{~ns}$ and $34.1 \mathrm{ns,}$ respectively. It was indicative of the positive effect of the MACl additive on the film crystallinity. Fig. $3 \mathrm{~B}$ is the normalized PL spectra of MAPI-MAAc and MAPI-BOAs films. Both samples show very similar PL peaks at around $779 \mathrm{~nm}$, but with very small blue shift from MAPI-BOAs to MAPI-MAAc sample. Such shift may be related to the crystal size or the Cl doping in the $\mathrm{MAPbI}_{3}$ in the films. It was also found that the MAPI-MACl sample has the largest band gap as shown in Fig. S5B. $\dagger$ Besides, the peak width at half height of MAPI-BOAs film is slightly reduced which indicates its lower band-edge trap state compared to MAPI-MAAc film. ${ }^{27}$ Furthermore, the $I-V$ response of the crystals in space-charge-limited-current (SCLC) regime is measured by the electron-only devices following the structure of $\mathrm{FTO} / \mathrm{TiO}_{2} /$ perovskite layer/PCBM/Ag, as can be seen in Fig. 3C and $\mathrm{D}$. At first stage of low voltage, the $I-V$ response is ohmic. When the voltage increases, the $I-V$ curve rises rapidly due to trap-filled limit (TFL), in which the injected charge carriers occupy all the trap states. ${ }^{28}$ The defect density is determined by the equation of $N_{\mathrm{t}}=2 \varepsilon \varepsilon_{0} V_{\mathrm{TFL}} / e L^{2}$, where $\varepsilon$ is the dielectric constant of $\mathrm{MAPbI}_{3}(=32), \varepsilon_{0}$ represents vacuum permittivity, $L$ is the thickness of perovskite layer ( $260 \mathrm{~nm}, \mathrm{Fig}$. S2 $\dagger), e$ is the elementary charge. The density of trap states is calculated to be $5.2 \times 10^{15}$ and $1.3 \times 10^{16} \mathrm{~cm}^{-3}$ respectively for MAPI-BOAs and MAPI-MAAc samples. The calculated trap state density of MAPbI-MACl is of $8.9 \times 10^{15} \mathrm{~cm}^{-3}$ (Fig. S6 $\dagger$ ), which is even a little higher than that of the MAPI-BOAs film possibly due to the presence of the hole structures. The relative large $N_{\mathrm{t}}$ of MAPI-MAAc sample should be attributed to the smaller size of MAPI-MAAc sample's crystals.

To further evaluate the performance of MAPI-BOAs perovskite, we then have measured its photoelectric properties. Fig. 4A shows the current $(J)$-voltage $(V)$ curves of the best performed device, which is measured under simulated AM 1.5 sunlight condition. The device's PCE is $17.0 \%$ at reverse scan, and $16.2 \%$ at forward scan with negligible hysteresis, which indicates the balanced charge transport in this PSC..$^{21,29}$ This should be associated with both the high quality and the growth orientation of perovskite crystals. For comparison, the MAPI-MAAc and MAPI-MACl PSCs were also measured and their devices performances were presented in Fig. S4. $\dagger$ The MAPI-BOAs deliver the highest efficiency. The reason why MAPI-MACl device, which has very similar crystal quality with MAPI-BOAs, show relative low PCE, is that the morphology of MAPI-MACl contains too much pin-holes, which aggravates the electron-hole recombination. The improvement of the $\mathrm{FF}$ value of the MAPI-BOAs PSC than the MAPI-MAAc based device is attributed to the reduced surface and the grain boundary defects as verified in Fig. 3. 


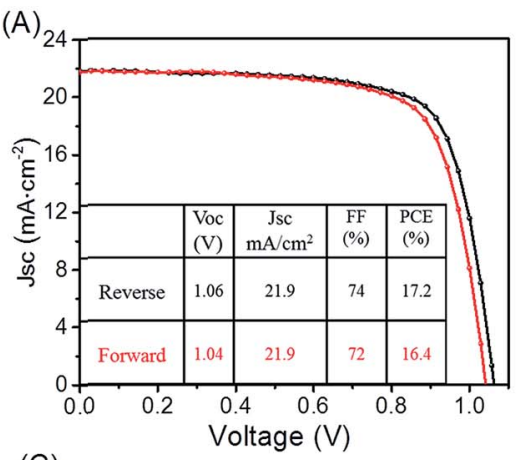

(C)

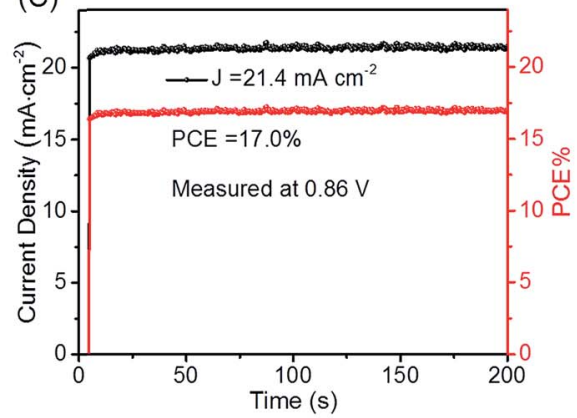

(B)

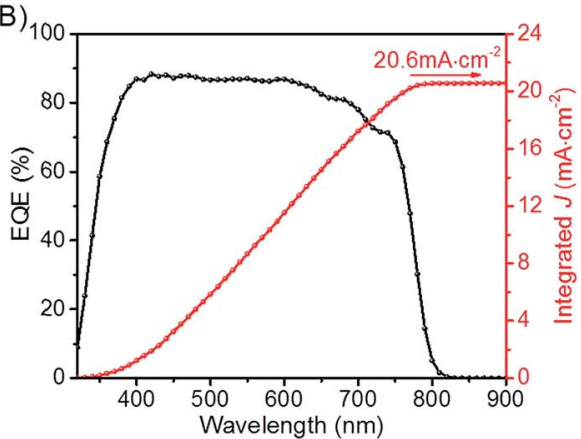

(D)

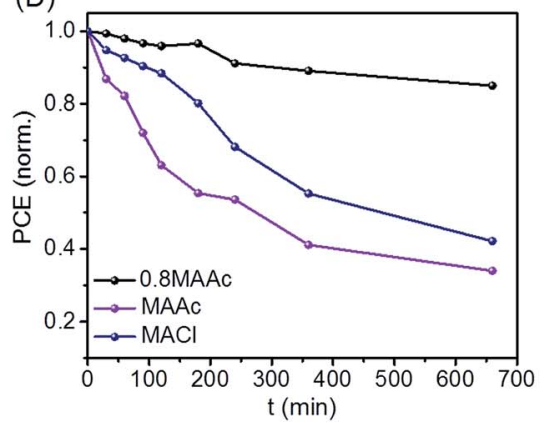

Fig. 4 (A) The J-V curves (reverse and forward scans) of the best MAPI-BOAs perovskite solar cells, (B) the corresponding EQE spectrum and integrated $\mathrm{J}_{\mathrm{sc}}$, and $(\mathrm{C})$ stabilized power output and current at maximum power point of MAPI-BOAs device under one-sun illumination. (D) Comparison of the stability of MAPI-MAAC, MAPI-BOAs and MAPI-MACI perovskite solar cells under continuous illumination at a relative humidity of $40 \%$.

Fig. $4 \mathrm{~B}$ is the external quantum efficiency (EQE) of the best devices and $88.3 \%$ incident photon-to-current efficiency is obtained. The integrated photocurrent density from the calculated photocurrent density of EQE spectrum is $20.6 \mathrm{~mA} \mathrm{~cm}^{-2}$, which is very close to the short circuit current density measured by us. Fig. 4C shows the superb stabilizing current/power output monitored by keeping the voltage at the maximum power point of $0.86 \mathrm{~V}$. The reproducibility of MAPI-BOAs devices is also counted in Fig. S4. $\dagger$ In Fig. 4D, we measure the illumination stability of the PSCs under simulated AM 1.5 sunlight irradiation. The stability comparison is tested under one sun illumination with a relative humidity of $40 \%$. During the interval of the $I-V$ measurements, the devices were kept at open circuit condition to speed up the degradation of the device. We can see that the MAPI-BOAs device shows superior stability compared to the others under illumination situation. All these excellent photoelectric properties confirm the high quality of MAPI-BOAs perovskite crystals and the optimized film morphology of devices.

\section{Conclusions}

In summary, we have reported an easy and promising strategy of using blended organic additives to regulate the crystal growing process of perovskite for obtaining a balance between film coverage and crystallinity. The nucleation and crystal growth processes are masterly observed in this work by in situ microscopy measurement, which gives inspiration for better understanding of the mechanism of additives. By simply incorporating $20 \% \mathrm{MACl}$ in MAAc additive, the morphology of the fabricated $\mathrm{MAPbI}_{3}$ film is modified at no expense of crystal crystallinity. The density of trap states is highly reduced due to the increase of the grain size. The PCE of the PSCs fabricated by this strategy is obviously enhanced in comparison the ones using single additive. This work also provides us with a promising strategy to use different blended additives with different properties for further application in other perovskite systems.

\section{Conflicts of interest}

There are no conflicts to declare.

\section{Acknowledgements}

Financial support for this work from the International S\&T Cooperation Program of China (2015DFG62670), National Natural Science Foundation of China (51672290, 21671196, 61604156), the Youth Innovation Promotion Association of CAS (2015167) and the Qingdao Key Lab of Solar Energy Utilization and Energy Storage Technology is gratefully acknowledged.

\section{Notes and references}

1 A. Kojima, K. Teshima, Y. Shirai and T. Miyasaka, J. Am. Chem. Soc., 2009, 131, 6050-6051.

2 W. S. Yang, B.-W. Park, E. H. Jung, N. J. Jeon, Y. C. Kim, D. U. Lee, S. S. Shin, J. Seo, E. K. Kim, J. H. Noh and S. I. Seok, Science, 2017, 356, 1376-1379. 
3 M. A. Green, A. Ho-Baillie and H. J. Snaith, Nat. Photonics, 2014, 8, 506-514.

4 K. Hwang, Y. S. Jung, Y. J. Heo, F. H. Scholes, S. E. Watkins, J. Subbiah, D. J. Jones, D. Y. Kim and D. Vak, Adv. Mater., 2015, 27, 1241-1247.

5 C. Li, Y. Zhou, L. Wang, Y. Chang, Y. Zong, L. Etgar, G. Cui, N. P. Padture and S. Pang, Angew. Chem., Int. Ed., 2017, 56, 7674-7678.

6 A. Polman, M. Knight, E. C. Garnett, B. Ehrler and W. C. Sinke, Science, 2016, 352, 307.

7 Z. Zhou, Z. Wang, Y. Zhou, S. Pang, D. Wang, H. Xu, Z. Liu, N. P. Padture and G. Cui, Angew. Chem., Int. Ed., 2015, 54, 9705-9709.

8 C. Li, S. Pang, H. Xu and G. Cui, Solar RRL, 2017, 1700076.

9 W. Nie, H. Tsai, R. Asadpour, J.-C. Blancon, A. J. Neukirch, G. Gupta, J. J. Crochet, M. Chhowalla, S. Tretiak, M. A. Alam, H.-L. Wang and A. D. Mohite, Science, 2015, 347, 522-525.

10 K. Yan, M. Long, T. Zhang, Z. Wei, H. Chen, S. Yang and J. Xu, J. Am. Chem. Soc., 2015, 137, 4460-4468.

11 C. Li, Z. Wang, Y. Chang, Y. Zong, F. Ji, B. Zhang, H. Li and S. Pang, RSC Adv., 2016, 6, 85026-85029.

12 R. L. Davidovich, V. Stavila, D. V. Marinin, E. I. Voit and K. H. Whitmire, Coord. Chem. Rev., 2009, 253, 1316-1352.

13 H. B. Kim, H. Choi, J. Jeong, S. Kim, B. Walker, S. Song and J. Y. Kim, Nanoscale, 2014, 6, 6679-6683.

14 W. Zhang, M. Saliba, D. T. Moore, S. K. Pathak, M. T. Horantner, T. Stergiopoulos, S. D. Stranks, G. E. Eperon, J. A. Alexander-Webber, A. Abate, A. Sadhanala, S. Yao, Y. Chen, R. H. Friend, L. A. Estroff, U. Wiesner and H. J. Snaith, Nat. Commun., 2015, 6, 6142.

15 D. Wang, Z. Liu, Z. Zhou, H. Zhu, Y. Zhou, C. Huang, Z. Wang, H. Xu, Y. Jin, B. Fan, S. Pang and G. Cui, Chem. Mater., 2014, 26, 7145-7150.

16 Y. Zhao and K. Zhu, J. Phys. Chem. C, 2014, 118, 9412-9418.
17 H. Yu, F. Wang, F. Xie, W. Li, J. Chen and N. Zhao, Adv. Funct. Mater., 2014, 24, 7102-7108.

18 Y. Xia, C. Ran, Y. Chen, Q. Li, N. Jiang, C. Li, Y. Pan, T. Li, J. Wang and W. Huang, J. Mater. Chem. A, 2017, 5, 31933202.

19 Q. Wu, P. Zhou, W. Zhou, X. Wei, T. Chen and S. Yang, ACS Appl. Mater. Interfaces, 2016, 8, 15333-15340.

20 Q. Wei, D. Yang, Z. Yang, X. Ren, Y. Liu, J. Feng, X. Zhu and S. Liu, RSC Adv., 2016, 6, 56807-56811.

21 W. Ke, C. Xiao, C. Wang, B. Saparov, H. S. Duan, D. Zhao, Z. Xiao, P. Schulz, S. P. Harvey, W. Liao, W. Meng, Y. Yu, A. J. Cimaroli, C. S. Jiang, K. Zhu, M. Al-Jassim, G. Fang, D. B. Mitzi and Y. Yan, Adv. Mater., 2016, 28, 5214-5221.

22 Q. Dong, Z. Wang, K. Zhang, H. Yu, P. Huang, X. Liu, Y. Zhou, N. Chen and B. Song, Nanoscale, 2016, 8, 55525558.

23 D. T. Moore, H. Sai, K. W. Tan, D. M. Smilgies, W. Zhang, H. J. Snaith, U. Wiesner and L. A. Estroff, J. Am. Chem. Soc., 2015, 137, 2350-2358.

24 G. Pellegrino, S. D'Angelo, I. Deretzis, G. G. Condorelli, E. Smecca, G. Malandrino, A. La Magna and A. Alberti, J. Phys. Chem. C, 2016, 120, 19768-19777.

25 S. Ahmad, P. K. Kanaujia, W. Niu, J. J. Baumberg and G. Vijaya Prakash, ACS Appl. Mater. Interfaces, 2014, 6, 10238-10247.

26 D. Shi, V. Adinolfi, R. Comin, M. Yuan, E. Alarousu, A. Buin, Y. Chen, S. Hoogland, A. Rothenberger, K. Katsiev, Y. Losovyj, X. Zhang, P. A. Dowben, O. F. Mohammed, E. H. Sargent and O. M. Bakr, Science, 2015, 347, 519-522.

27 J. A. Carr and S. Chaudhary, Energy Environ. Sci., 2013, 6, 3414-3438.

28 Z. Liu, J. Hu, H. Jiao, L. Li, G. Zheng, Y. Chen, Y. Huang, Q. Zhang, C. Shen, Q. Chen and H. Zhou, Adv. Mater., 2017, 29, 1606774.

29 N. Vaenas, D. Konios, T. Stergiopoulos and E. Kymakis, RSC Adv., 2015, 5, 107771-107776. 